\title{
Quick-staining of whole retina for pathological demonstration of cystoid macular edema
}

\author{
J. Reimer Wolter \\ The Departments of Ophthalmology and Pathology of the University of Michigan Hospitals, Ann Arbor, Michigan, USA
}

\begin{abstract}
Quick-staining of the whole central retina with Hematoxylin-Eosin allows for a pathological demonstration of cystoid macular edema (CMC) that closely resembles its clinical fluorescein angiography appearance. Use of the simple new method will result in a better understanding of occurrence, patterns, stages and extent of the cystoid changes as well as of the associated central folding in $\mathrm{CME}$ under different clinical conditions. The preservation of a central vision around $20 / 40$ in the present case with distinct CME is emphasized.
\end{abstract}

Zusammenfassung. Schnellfärbung der zentralen Netzhaut in ganzer Dicke mit Haematoxylin-Eosin erlaubt eine pathologische Darstellung von zystoidem Makulaoedem, die dem klinischen Bild bei Fluorescein Angiographie sehr ähnlich ist. Anwendung dieser einfachen neuen Methode wird einen besseren Einblick in das Auftreten, die Anordnung, die Phasen und die Ausdehnung sowie die associierte Faltenbildung bei zystoidem Makulaoedem unter verschiedenen klinischen Bedingungen geben. Ein erhaltenes zentrales Sehvermögen von etwa 6/12 in dem beschriebenen Fall von cystoidem Makulaoedem ist bemerkenswert.

\section{Introduction}

To bridge the gap between ophthalmoscopic picture and pathological observation in the field of retinal diseases is a constant struggle for the clinically oriented eye pathologist. This struggle not only aims to help the clinician in the understanding of the message in a pathological specimen, but it primarily wants to overcome the investigators own problems. It is very difficult, for example, to reconstruct the arrangement of the cystoid spaces of cystoid macular edema (CME) branching in the plane of the central retina from serial cross sections and to understand its structure in relation to the surrounding retinal components. The facts that many retinal formations are arranged parallel to the flat surfaces of this film-like layer and that routine sections of the eye show this thin layer in cross section greatly contribute to this problem.

\footnotetext{
* Supported by The Research To Prevent Blindness, Inc., New York, N.Y., USA

Offprint requests to: Reimer Wolter, M.D., Department of Ophthalmology, University of Michigan Hospitals, Ann Arbor, Michigan 48109 , USA
}

The star-like branching pattern of early CME is very familiar to all clinicians from the study of fluorescein angiograms. There is a great need for a simple technique that allows for the demonstration of this branching pattern in a pathological specimen. To present such a technique is the purpose of the present paper. A case of typical CME in an eye with a choroidal melanoma is used as an example to demonstrate the new staining method and its potentials.

\section{Case report}

This 66 year-old female was seen in consultation by Dr. Andrew K. Vine of this Ophthalmology Department on 12.9.82. The patient had observed slowly progressive vision loss in the left eye within the last year. Vision was 20/40 in the right eye and $20 / 50$ in the left with glasses. A pinhole allowed for improvement to $20 / 30$ in the right eye, but it did not help in the left. The left eye exhibited a solid choroidal mass of brownish color in the superior midperiphery. This mass did not transilluminate and it was clinically compatible with a choroidal melanoma. This clinical diagnosis was supported by ultrasound studies. Some central retinal degeneration with diffuse macular drusen was seen in both eyes. A diagnosis of a choroidal melanoma in the left eye was made. Observation was recommended and it was stated that enucleation would be indicated, if progressive growth of the melanoma could be clinically demonstrated.

The referring physician, Dr. William Arendshorst of Holland, Mich., followed the patient clinically. The patient requested enucleation and this was done by Dr. A. without difficulties on 12.30 .82 . The globe was fixed in formalin immediately after enucleation. For the present study it is of the greatest importance to state that this left retained a central vision of about 20/40 up to the time of its enucleation.

The eye ball was of normal size and did not show extraocular tumor extension. Transillumination revealed the shadow of the tumor superiorly. The eye was opened in a vertical plane with a cut along the nasal spects of the disk and the choroidal mass. This mass was oval in shape, measured $9 \times 7 \times 2^{1 / 2} \mathrm{~mm}$ and had a gray-brown color. Histological sections made in this vertical plane (Fig. 1) revealed a normal cornea, anterior chamber, iris and lens (1). The ciliary body showed infiltration with occasional mononuclear inflammatory cells. The vitreous (v) showed syneresis and slight detachment (d) of the posterior vitreous face in an area posterior to the choroidal tumor (d). The vitreous 


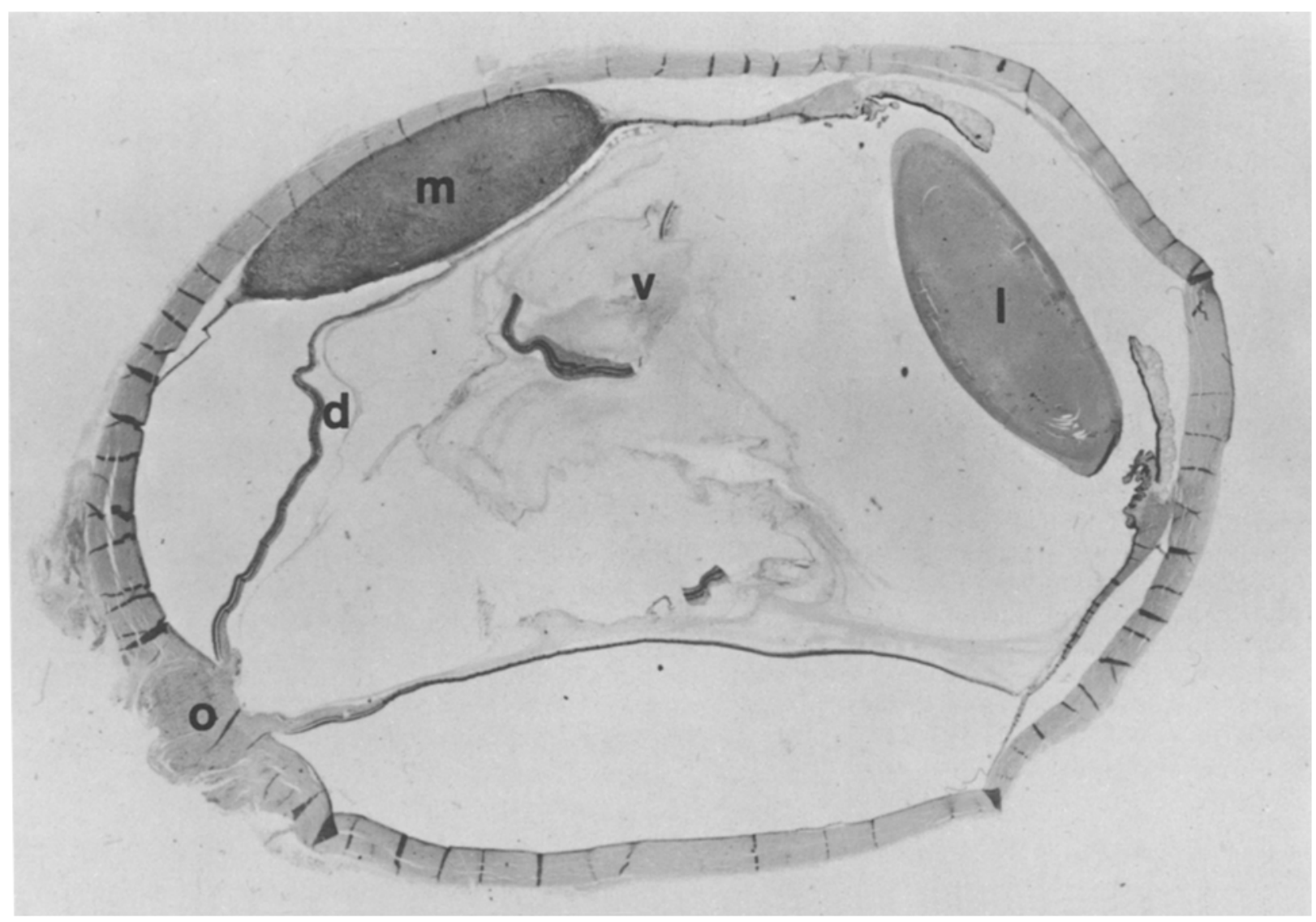

Fig. 1. Vertical cross section of the present eye showing the lens $(l)$, the melanoma $(m)$ in the superior choroid, the vitreous $(v)$ with a few artificially displaced pieces of retina, some early detachment of the posterior vitreous face $(d)$, and the optic nerve $(o)$. - Paraffin section, $\mathrm{H}$ and $\mathrm{E}$ stain, photomicrograph $\times 7$

was firmly attached to the optic nerve (o). The tumor (m) had no extensions through Bruch's membrane nor into the sclera. It was mainly composed of spindle shaped cells with nucleolated nuclei, but groups of more atypical cells of epithelioid shape and with large nuclei as well as frequent mitoses were seen. The diagnosis of a malignant choroidal melanoma of an epithelioid type without direct extraocular extensions was made.

The foveal retina was separated from the firmly attached vitreous and the remainder of the ocular wall in a rectangular piece of retina measuring $7 \times 9 \mathrm{~mm}$. With a glass rod this piece of whole retina was transferred to running water and washed for 10 minutes. Next, it was placed with the same glass rod step by step in the following solutions: Hematoxylin stain for $30 \mathrm{sec}$, water for rinsing $10 \mathrm{sec}$, Eosin stain for $30 \mathrm{sec}, 96 \%$ and $100 \%$ alcohol $20 \mathrm{sec}$ each for dehydration and in Xylol $60 \mathrm{sec}$ for final clearing. From the Xylol the piece of retina was placed onto a glass slide covered with a layer of embedding fluid. For the present study the retina was turned upside down with the rods and cones pointing up. More embedding fluid was dropped onto the piece of retina and a cover glass was placed on top of this. The whole process takes less than $10 \mathrm{~min}$ and the specimen can be taken to the microscope for study and photography as soon as the cover glass has been placed.

The view of the foveal retina under the microscope in the present specimen was in its appearance surprisingly similar to well developed CME in the living eye after fluorescein angiography (Fig. 2). The foveola was replaced by a clear cyst (arrow). Extending horizontally from this clear cyst to both sides there were dichotomously branching cystoid channels and small isolated-appearing cystoid formations. These channels and small isolated cysts were more extensive in the direction towards the disk (d) as compared to the cystoid formations pointing temporally from the foveola. Higher microsocpic power showed the cystoid spaces to be near the suface of the specimen (Figs. 3 and 4). The rods with their outer segments were visible on top and there was a distinct fold formed by the cone segments seen to extend from the foveola (f) in both horizontal directions towards the branching cystoid spaces slightly deeper in the retina. In the region of the cystoid foveola the cones were absent (Fig. 4).

\section{Discussion}

Progress often starts out with a new tool or a new technique - especially when this is simple, cheap and not very timeconsuming. The present method can be used by anybody with the equipment present in any pathology laboratory for routine staining. The quickness of the staining process is essential for the best possible contrast between normal retinal tissues and the fluid-filled cystoid spaces. Most of the fluid in these spaces is not sufficiently proteinanceous to be fixed by formalin [1]. The trick is to stop the staining process, after the stains have done their work in the retinal tissues - and before they have a chance to leak into the 


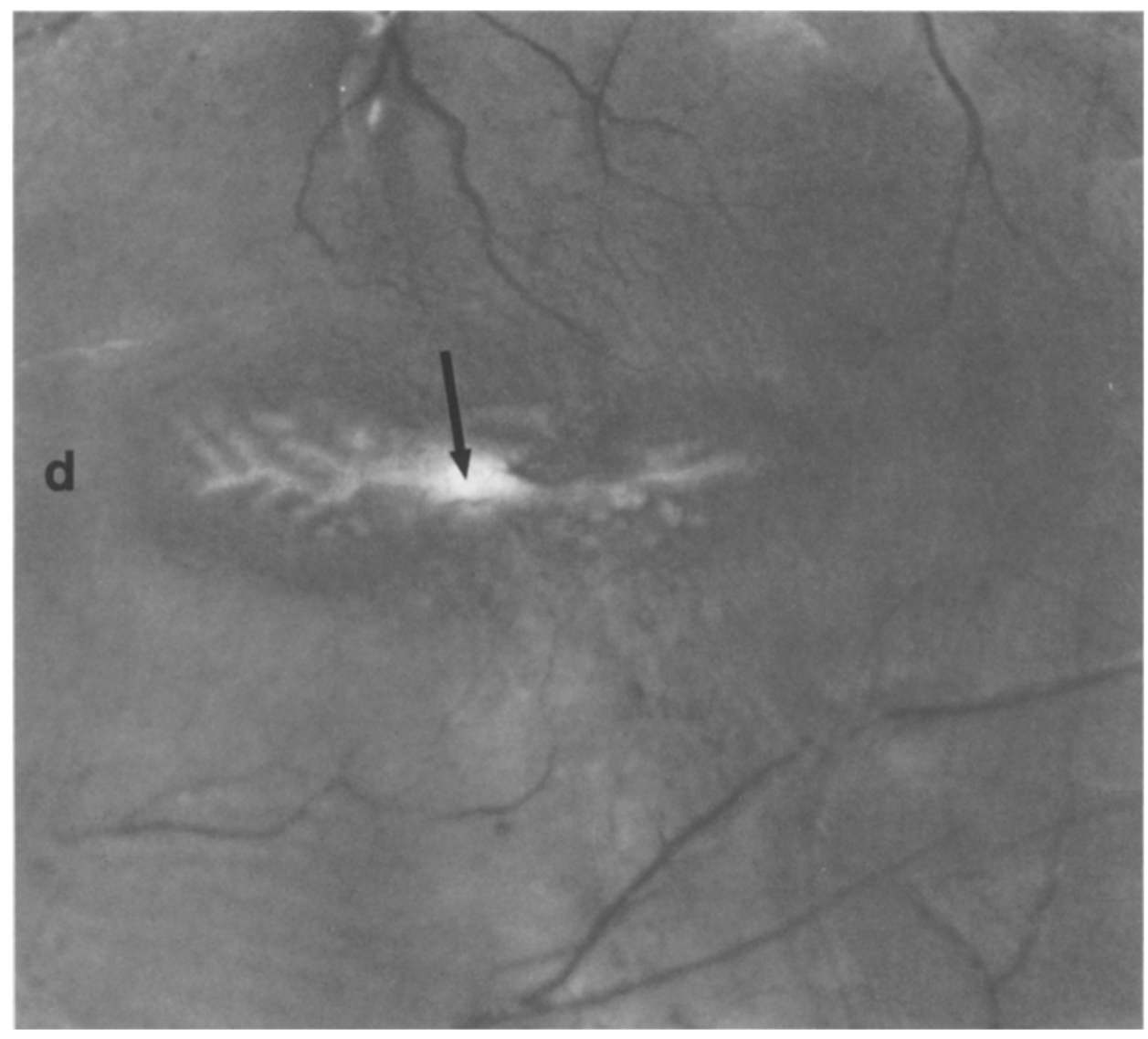

Fig. 2. View of the fovea with parafoveal blood vessels and the cystic foveola (arrow) in the center. Extending from the foveola horizontally to both sides there are the branching patterns of cystoid spaces. These are best developed towards the disk $(d)$. - Quick-stain of whole retina with $\mathrm{H}$ and $\mathrm{E}$, photomicrograph $\times 25$

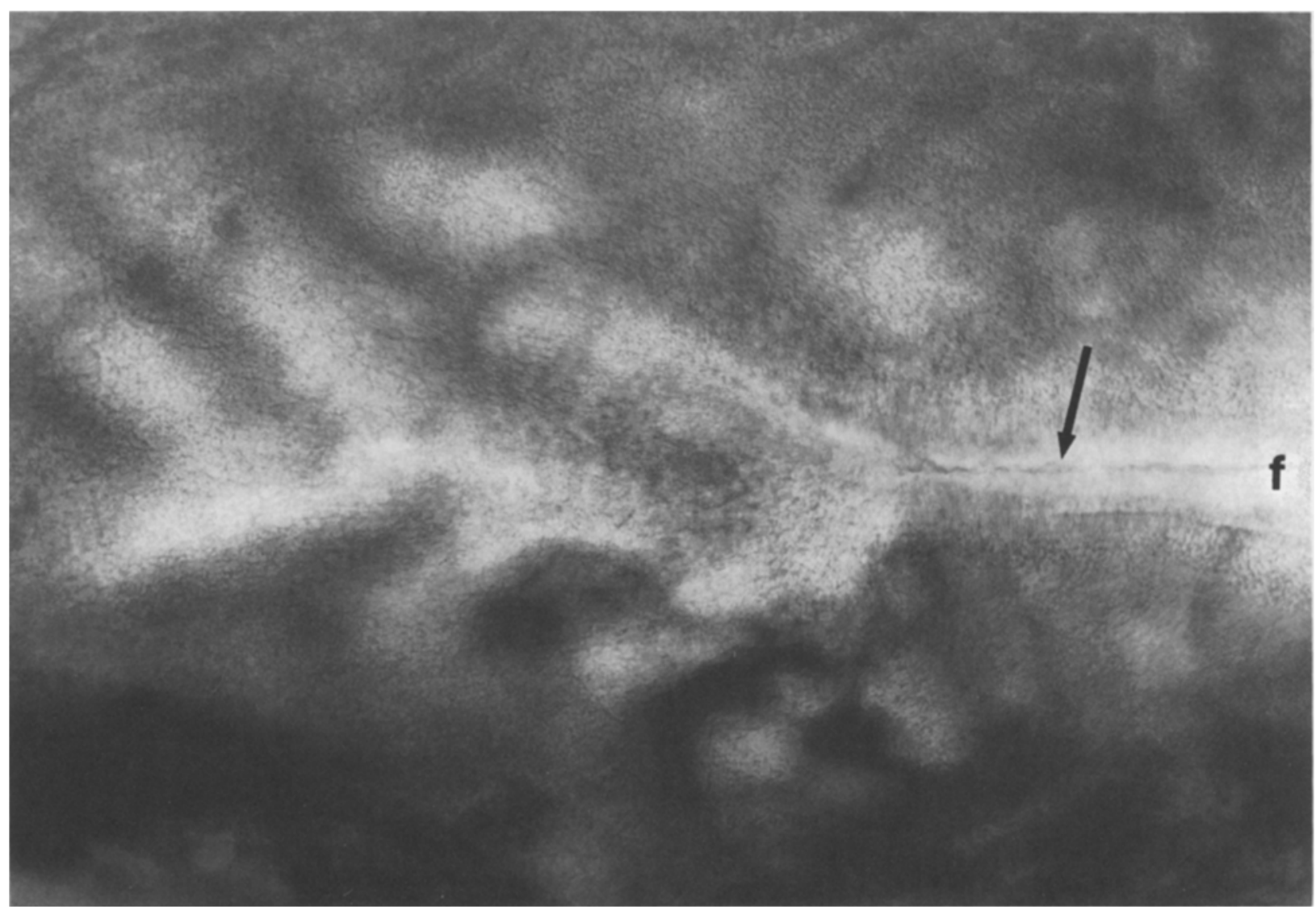

Fig. 3. Higher power of the unstained channels and cystoid spaces extending from the cystic foveola $(f)$ in a nasal direction towards the disk. A horizontal fold (arrow) is seen to involve the cones and this runs towards the disk - in the main direction of the channels and spaces. - Quick-stain of whole retina with $\mathrm{H}$ and $\mathrm{E}$, photomicrograph $\times 125$ 


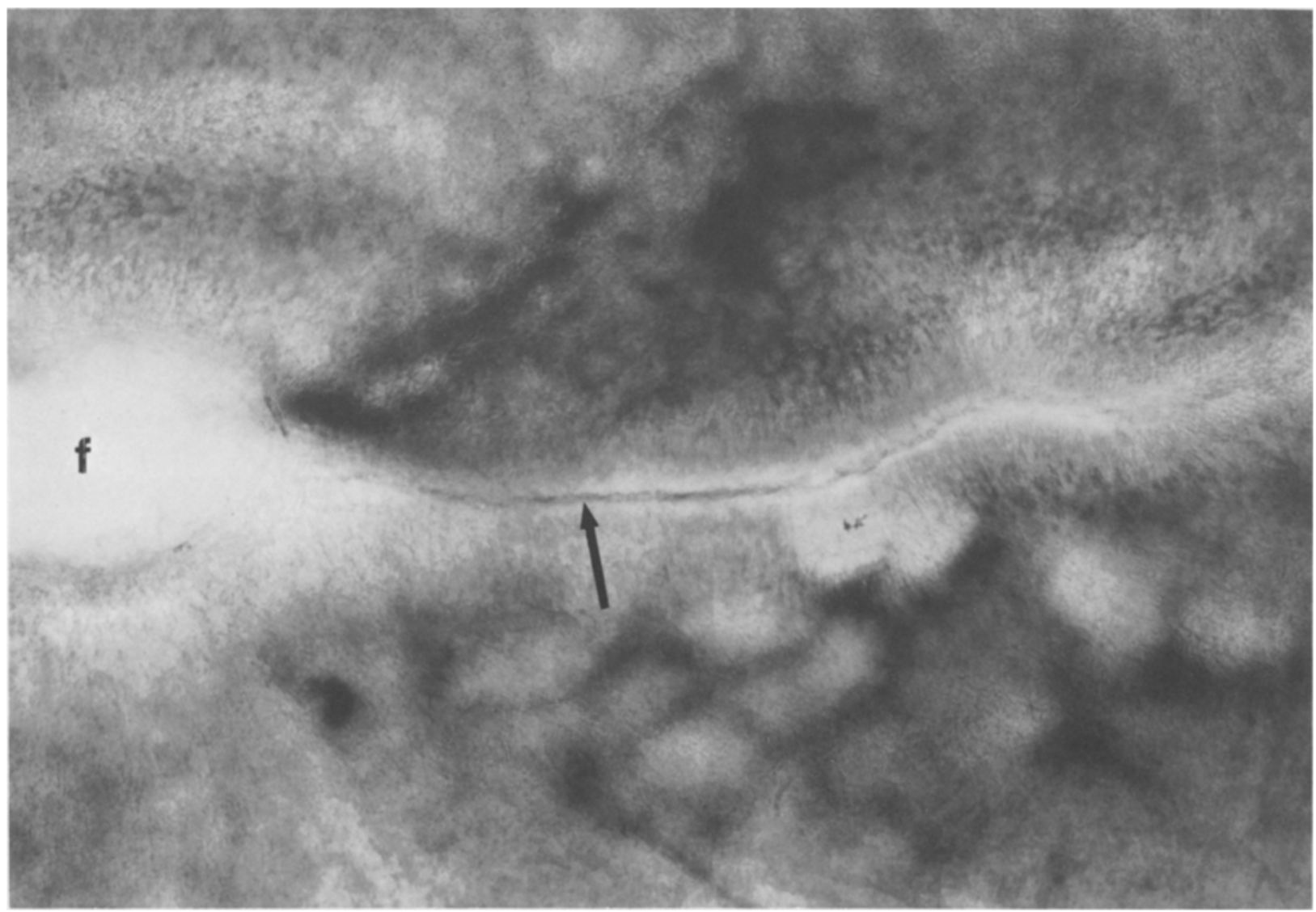

Fig. 4. Higher power of the foveola $(f)$ and the temporal extensions of the unstained channels and cysts with another horizontal fold mainly formed by cones (arrow). - Quick-stain of whole retina with $\mathrm{H}$ and E, photomicrograph $\times 125$

fluids of the cystoid spaces. The fact that the walls of the cystoid spaces present a definite barrier to the leakage of the dyes is of interest and may well give support to the view that these spaces are not cystoid after all, but that they have a wall formed by the processes of retinal glia. Only this is not seen in routine stains. The contrast between the clear cystoid spaces and the surrounding retinal tissues is lost, when the retina is left in the stains too long.

The low-power microscopic view of the central retina with CME after the quick-staining method described here certainly resembles the typical fundus view of CME in a fluorescein angiogram. In the present case the foveola is changed into a cystoid space and the parafoveolar spaces appear to be related to this central space in a typical pattern of branching channels and smaller spaces. By location these spaces are seen to be directly adjacent to the outer nuclear layer at the level of the outer plexiforme layer. The central retina is distincly thickened due to the cystoid changes and the foveola presents as a small mound and not as a valley. A distinct fold of paracentral cones and their outer segments running horizontally with the cystic foveola as its center is observed. Folds like this have been described before in association with CME [1-9] and it seems that they greatly vary in their direction and depth under different conditions. One fold of the outer retina that resembles the fold seen in the present case was presented in a paper on foveal involvement in choroidal melanomas in this Archive in 1982 [1], Figs. 6 and 7. All folds of this type are associated with some separation of the cones from the underlying pigment epithelium of the choroid and may, thus, be directly responsible for some visual disturbance. The relationship of the slight separation of central cones from the pigment epithelium associated with this folding of outer retinal layers to the socalled pseudophakic serous maculopathy [10] is not as yet clear.

The fact that the present patient had a central vision of between $20 / 40$ and $20 / 50$ in the involved eye up to the time of its enucleation supports my earlier observation that the this kind of vision certainly is compatible with the presence of rather well developed and active CME [2]. There is reason to reemphasize this clinical fact, because there is a trend among lens implantation surgeons to list all cases with postoperative vision between $20 / 20$ and $20 / 40$ as clinically successful and uncomplicated. In view of my observations, a postoperative central vision of $20 / 40$ certainly does not exclude the possibility of existing active CME.

It is very important to point out, finally, that the quickstained piece of retina can be removed again from the slide, after it has been studied and photographed, by placing the whole slide with its coverglass in Xylol. When the embedding fluid is dissolved, the retina can be embedded in paraffin for serial sectioning on a microtome. This allows for high-power demonstration of histological details in the same tissues that were seen before at low power in the flat preparation.

The single present case does not allow for any conclusions. To describe the possibility of the demonstration of $\mathrm{CME}$ with whole stains of the central retina is the purpose 
of this paper. It is hoped that this will be done in many cases by many investigators for a better understanding of the basic pattern, occurrance and extend of $\mathrm{CME}$ in relation to ocular and general disease processes.

\section{References}

1. Wolter JR (1981) The histopathology of cystoid macular edema. Graefe's Arch Clin Exp Ophthalmol 216:85-101

2. Wolter JR, Croasdale RE, Bahn ChF (1980) Reactions to an anterior chamber lens - two years after implantation. Ophthal Surg 11:794-800

3. Wolter JR (1981) Vertical folds of central retina and choroid in sudden ocular decompression. Ophthal Surg 12:190-194

4. Wolter JR (1981) Foveal pathology following anterior segment injury. Ophthal Surg 12:661-665
5. Wolter JR (1982) Foveal pathology in ciliary body melanoma. Ophthal Surg 13:309-311

6. Wolter JR (1982) Three basic types of foveal involvement in choroidal melanomas. Graefe's Arch Clin Exp Ophthalmol 218:237-243

7. Wolter JR (1982) Concentric microwaves of Henle's fiber layer in association with cystoid macular edema Graefe's Arch Clin Exp Ophthalmol 219:1-5

8. Wolter JR (1982) Leaky cystoid macular edema attracting vitreous macrophages. Ophthal Surg 13:568--571

9. Wolter JR (1982) Expulsive hemorrhage: a study of histopathological details. Graefe's Arch Clin Exp Ophthalmol 219:155-158

10. Macy JI, Baerveldt G (1983) Pseudophakic serous maculopathy. AMA Arch Ophth 101:228-231

Received February 23, 1983 